\title{
Value-Centric Design Architecture Based on Analysis of Space System Characteristics
}

DOI:

10.1016/j.actaastro.2017.12.017

\section{Document Version}

Accepted author manuscript

Link to publication record in Manchester Research Explorer

\section{Citation for published version (APA):}

Xu, Q., Hollingsworth, P., \& Smith, K. (2018). Value-Centric Design Architecture Based on Analysis of Space System Characteristics. Acta Astronautica, 144, 69-79. https://doi.org/10.1016/j.actaastro.2017.12.017

\section{Published in:}

Acta Astronautica

\section{Citing this paper}

Please note that where the full-text provided on Manchester Research Explorer is the Author Accepted Manuscript or Proof version this may differ from the final Published version. If citing, it is advised that you check and use the publisher's definitive version.

\section{General rights}

Copyright and moral rights for the publications made accessible in the Research Explorer are retained by the authors and/or other copyright owners and it is a condition of accessing publications that users recognise and abide by the legal requirements associated with these rights.

\section{Takedown policy}

If you believe that this document breaches copyright please refer to the University of Manchester's Takedown Procedures [http://man.ac.uk/04Y6Bo] or contact uml.scholarlycommunications@manchester.ac.uk providing relevant details, so we can investigate your claim.

\section{OPEN ACCESS}




\title{
Value-Centric Design Architecture Based on Analysis of Space System Characteristics ${ }^{\text {th }}$
}

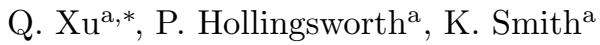 \\ ${ }^{a}$ School of Mechanical, Aerospace and Civil Engineering, The University of Manchester, George Begg Building, Sackville St, Manchester, \\ M13 $9 P L$
}

\begin{abstract}
Emerging design concepts such as miniaturisation, modularity, and standardisation, have contributed to the rapid development of small and inexpensive platforms, particularly cubesats. This has been stimulating an upcoming revolution in space design and development, leading satellites into the era of "smaller, faster, and cheaper". However, the current requirement-centric design philosophy, focused on bespoke monolithic systems, along with the associated development and production process does not inherently fit with the innovative modular, standardised, and mass-produced technologies. This paper presents a new categorisation, characterisation, and value-centric design architecture to address this need for both traditional and novel system designs. Based on the categorisation of system configurations, a characterisation of space systems, comprised of duplication, fractionation, and derivation, is proposed to capture the overall system configuration characteristics and promote potential hybrid designs. Complying with the definitions of the system characterisation, mathematical mapping relations between the system characterisation and the system properties are described to establish the mathematical foundation of the proposed value-centric design methodology. To illustrate the methodology, subsystem reliability relationships are therefore analysed to explore potential system configurations in the design space. The results of the applications of system characteristic analysis clearly show that the effects of different configuration characteristics on the system properties can be effectively analysed and evaluated, enabling the optimization of system configurations.
\end{abstract}

Keywords: Conceptual design, Value-centric design, System characterisation, Configuration formulation

\section{Introduction}

Emerging technologies, such as widely-used microtechnology and nanotechnology derived from the electronics industry, offer significant opportunities for the miniaturisation of space systems[1]. The concept of modularity associated with commercialisation not only completely changes the labour-intensive and bespoke situation of the existing space industry by promoting their standardisation and reconfigurability[2], but also obviously drives the reduction of design, integration and testing time and associated costs[3]. Therefore, these innovative concepts and technologies have contributed to the rapid development of small and inexpensive space platforms, e.g., cubesats and nanosats, stimulating an upcoming revolution in space design and development.

New design concepts have also promoted a big breakthrough in space system capabilities, some even overturning the conventional understanding of space. Duplicated

\footnotetext{
This paper was presented at the International Astronautical Congress in 2016 in Guadalajara, Mexico.

* Corresponding author.

Email addresses: qin.xu-2@manchester.ac.uk (Q. Xu), peter.hollingsworth@manchester.ac.uk (P. Hollingsworth), kate.smith@manchester.ac.uk (K. Smith)
}

configurations are capable of broadening the range of space missions and simultaneously reducing the design requirements of individual units to some extent. Distributed systems, which refer to a cluster of satellites cooperating as a virtual satellite in the form of spatial separation, with each sharing the communication, processing and payload[4], have offered an excellent carrier for modularity, mass production and the use of the Commercial Off-The-Shelf (COTS) products. Such a configuration is believed to be flexible, robust and cost-effective throughout the lifecycle[5], filling the vacancies of flexibility and robustness in traditional systems and creating additional values to the systems.

However, current design, production and certification methodologies generally spend lots of time and money on the integration and testing of large, complex and customised systems $[6,7,8]$, which do not inherently fit with the modular, standardised, and mass-produced characteristics of these innovative concepts. Since "smaller, faster, and cheaper" $[9,10,11]$ has been one of the latest driving factors for spacecraft design, new analysis and design methodologies are urgently required to deliver such capabilities effectively. Therefore, a new insight for future space mission design and analysis will be proposed in this paper. To describe and analyse space systems diversely 
and comprehensively, a new architecture for the design and development of a wide range of space systems needs to be developed, enabling the applications of both traditional and innovative concepts and technologies.

More specifically, such an architecture needs to realise both the traditionally qualitative analysis on different mission characteristics and the innovatively quantitative design on system configuration characteristics. One of the predominant advantages of such a new design paradigm is enabling the application of various optimization methods to explore the system configuration tradespace for the optimal solution, with system properties or system value models as objective functions, which can evaluate system property or value associated with its variance and probabilistic distribution of unanticipated events.

\section{System Configuration Characteristics}

Based upon the categorisation of different spacecraft, the system characteristic space consisting of degree of duplication, fractionation, and derivation is proposed. Their definitions and characteristics are illustrated for a qualitative analysis of mission characteristics. Consequently, the connection between mission concept design and system characteristics is established, enabling the mission concept exploration.

\subsection{Categorisation of Space Systems}

Space systems are classified into four categories: monolithic spacecraft, constellations of identical spacecraft, fractionated spacecraft and hybrid spacecraft, with the definition and characteristics of each category clarified as follows.

Monolithic spacecraft, also known as traditional spacecraft or singular spacecraft, are generally space systems comprising of only one satellite that has a monolithic configuration, where functionally independent subsystems, such as data handling, communication and power, are not architecturally separated but integrated on a single physical platform. In essence, a monolithic system refers to a unchangeable structure without any individual variation, so that it may be powerful but slow to change[12].

For monolithic spacecraft, all the mission objectives are realised by a single spacecraft itself. Any component failure on board may weaken the integrity of the entire system, and endangers overall system capability and lifecycle value. Despite cautious selection of components, duplication design, and excessive ground testing, fragility remains in such a single and tightly coupled system[13], which is an intrinsic attribute of traditional design.

Monolithic spacecraft can accomplish a variety of missions, ranging from earth observation to interplanetary exploration, almost all the known missions. Examples are the first artificial satellite Sputnik-1[14] , the first interstellar spacecraft Voyager-1[15], the National Aeronautics and Space Administrations (NASA) asteroid probe Dawn[16]

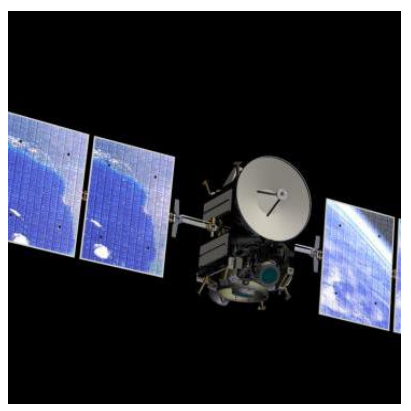

Fig. 1: Dawn[16]

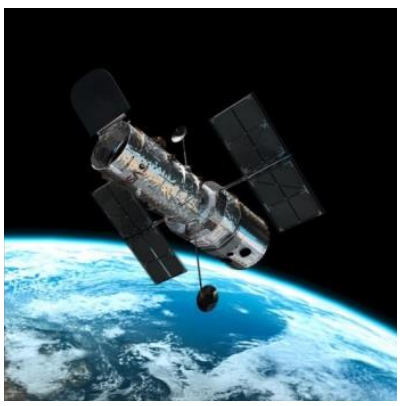

Fig. 2: $\operatorname{HST}[18]$ seen in Fig. 1, the largest and most complex on-orbit telescope Hubble Space Telescope (HST)[17] shown in Fig. 2.

Constellations of identical spacecraft transform monolithic spacecraft into homogeneous clusters composed of a group of identical or near identical spacecraft flying in a certain formation or constellation, which can function independently from each other[19]. They are commonlyused in near-Earth space that requires a continuous and global service. Additionally, a series of identical spacecraft are likely to be manufactured in production lines[20] and can therefore reduce the scale of costs.

Unlike monolithic spacecraft, which operate alone throughout the entire lifetime, constellations of identical spacecraft tend to work in-group with necessary maintenance or upgradation. Apart from the initial launched space system, a set of replenishment satellites are often developed in case of any orbit failure or system upgrade. As the project progresses, a series may be replaced by a more competent or economical series, for the purpose of improving the system capabilities or extending the mission operations. Moreover, losing any satellite should not destroy the entire service for well-designed constellations, at most functionality degradation to some extent.

Examples here consist of the famous navigation constellation the Global Positioning System (GPS) shown in Fig. 3, which achieved its Full Operational Capability (FOC) in 1995 and have been undertaking replenishment and modernized processes since 2005[21], the European Global Navigation Satellite System (GNSS) Galileo seen in Fig. 4 , the unsuccessful commercial communication constellations in the Low Earth Orbit (LEO) such as Iridium, and the Tacking and Data Relay Satellites (TDRS)[22] in the Geostationary Earth Orbit (GEO).

Fractionated spacecraft decompose monolithic spacecraft into heterogeneous clusters of wirelesslyinterconnected modules, each capable of sharing and utilizing resources throughout the entire network[6, 25, 26]. Unlike constellations of identical spacecraft, where each single spacecraft in a cluster is identical or near identical, almost every module of fractionated spacecraft has at least one distinctive functionality[27], corresponding to various subsystems of a monolithic system.

As DARPA describes[28], such architecture enhances the adaptability and survivability of space systems, 


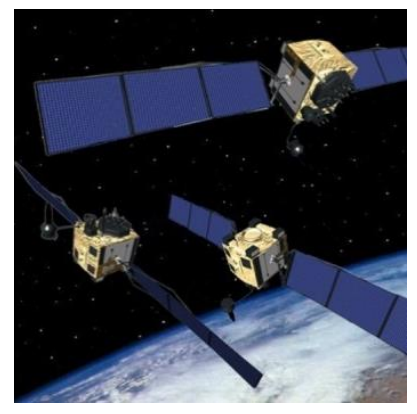

Fig. 3: GPS Block IIF[23]

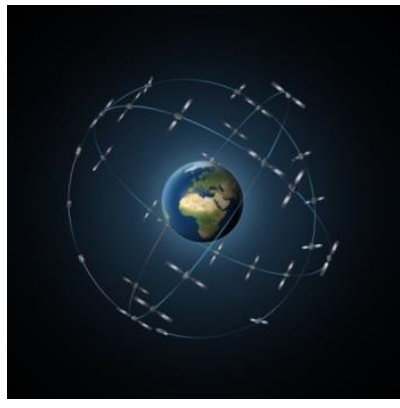

Fig. 4: Galileo[24]

while shortening development timelines and reducing the barrier-to-entry for participation in the security space industry. Flexibility comes from the fractionation of functionalities, making the entire system more robust to any unforeseen damage or failure. Meanwhile, modularity and commoditization cut down the cost and development cycle. Thus, fractionated spacecraft are considered as an emerging concept with promising applications.

To date there exist no true operational fractionated spacecraft. The systems currently only exist as conceptual and technological examples such as System F6 developed by DARPA[29] seen in Fig. 5, described as Future Fast, Flexible, Fractionated, Free-Flying Spacecraft united by Information eXchange.

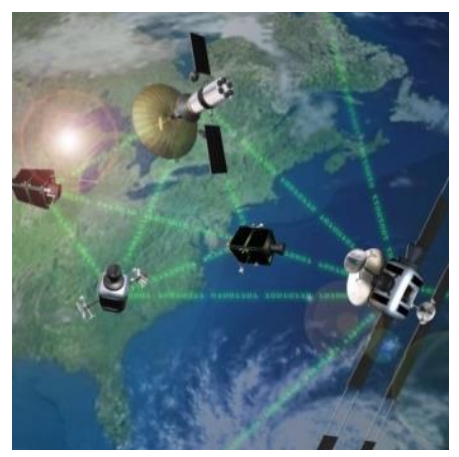

Fig. 5: System F6[28]

The remainder of space systems are considered as hybrid spacecraft, namely the combinations of constellations of identical spacecraft and fractionated spacecraft. Strict to the definition, each member of a fractionated spacecraft is different, while backups are quite common in spacecraft design. Thus, pure fractionated spacecraft only exist theoretically, and hybrid spacecraft can be practically merged with fractionated spacecraft.

\subsection{System Characteristic Space}

The system characteristic space is constructed by three different dimensions, which are degree of duplication, fractionation, and derivation, shown in Fig. 6. These three characteristics represent different system properties and capabilities. Duplication is a common approach to directly increase system reliability, which in turn manages mission

uncertainties and risks effectively. Fractionation is an innovative design concept proposed to shorten the design and development cycle of coupled subsystems, lessen individual launch mass and capacity, and reduce maintenance and upgrade cost. Derivation draws on the experience of previous design paradigms to largely cut down the risk and the time of a design and development process, enabling a responsive and inexpensive design.
Hubble

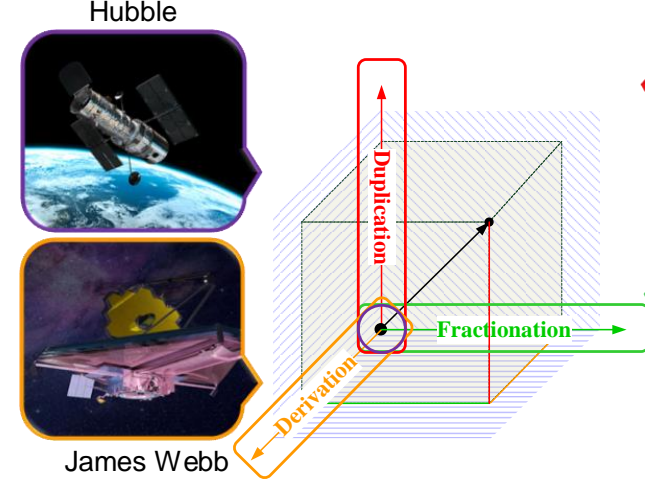

GPS

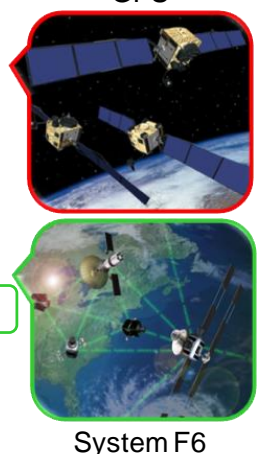

Fig. 6: System Characteristic Space (The colour purple, red, green, and yellow match the monolith, duplication, fractionation, and derivation respectively.)

Duplication refers to components, subsystems, or satellites in a system, technically duplicated to realise more powerful functions or in case of on-orbit failures. Within the domain of duplication, each unit performing the same function can be exchanged by another one. Duplication, also known as redundancy, is widely used in system engineering with the intention of increasing the reliability of a system. Such duplication is generally integrated, while the proposed duplication can be spatially distributed in different units of a space system, instead of being limited to the monolithic configuration.

On the other hand, duplication is necessary in space missions such as GPS and TDRS since individuals cannot implement the FOC. Currently, 31 of the 32 satellites in the GPS constellation are active, with on average 9 satellites visible from any point on the ground at one time[30], ensuring the minimum 4 satellites for any position calculation[31]. Of the 9 satellites, 4 are duplicated to meet the FOC, while the other 5 are used for improving the calculation accuracy or redundancy.

Fractionation refers to the spatial distribution of the essential functionalities in a system. The major difference between fractionation and duplication is that fractionation increases the heterogeneous degree of a space system, while duplication increases its homogeneous degree. Unlike duplication, each member in a fractionated system performs different functions to fulfill the collaborative mission, without any one missing.

Previous studies[19, 32, 33] have reported that networking, wireless communication, cluster flight, and distributed computing are the four critical technologies to realise the fractionation of space systems. As one of the biggest char- 
acteristics of current space technologies, the incremental use of on-board processing[34] offers a great opportunity for the application of fractionation. The fractionation of such subsystems as attitude and orbit control subsystem (AOCS) may stimulate the decrease of its own scale, for only certain subsystems need to be controlled instead of the entire spacecraft. Different from the accurate orbit maintenance of traditional constellations, the cluster flight of fractionated spacecraft only requires appropriate relative distances and orientations to ensure communication links and collision avoidance[35].

Derivation refers to components, subsystems, or satellites in a system, whose technologies and capabilities are derivative from previous missions. On one hand one could say that, the more derivative elements in a system, the more mature a system is, which may imply higher reliability and cost-effectiveness of the entire system. However on the other hand, the innovative or less derivative products with advanced design concepts and technologies may extend system capability or improve system performance.

\subsection{Mission Characteristic Analysis}

The proposed system characteristic space can act as a valuable tool for both qualitative and quantitative analysis of space mission design. In this section, the qualitative analysis of space mission characteristics is performed, while the rest of this paper will focus on the quantitative analysis of space system concept design.

According to the definitions of system characteristic space, different space missions can be evaluated and compared. Taking a series of classical space missions as examples, e.g., Mariner \& Voyager (M\&V), Hubble Space Telescope (HST), Global Positioning System (GPS), International Space Station (ISS), and RapidEye (RE), an exemplary assessment of different mission configuration characteristics was carried out, seen in Fig. 7, where the levels from the lowest to the highest represent the duplication/fractionation/derivation levels are achieved from none (level 1), component (level 2), subsystem (level 3), satellite (level 4) to system level (level 5) respectively.

Mariner was a space program consisting of a series of interplanetary probes designed for investigating various planets in the solar system, with Voyager program as its extension. As Voyager 1 and 2 were almost identical, Mariner series also had the architecture of redundancy, e.g., Mariner 3 and 4 were identical teammates in the mission of Mars flyby. Moreover, the Mariner series were derivative in both mission and technical view. As spacecraft design and development technology got more and more advanced, space mission went further and further, from Venus to Neptune, from interplanetary space to interstellar space. For instance, Mariner 3 and 4 were just Mars flyby missions, while Mariner 8 and 9 were designed to map the Martian surface.

Monolithic architecture is believed to be one of the most applicable and reliable solutions for current outer space traveling, while duplication and fractionation may bring about unpredictable risks to the mission. Many examples have proved the advantages of monolithic configuration, such as voyager-1, which is the first interstellar spacecraft in the history of space exploration. Although fractionation may provide additional survivability, such technical difficulties as interplanetary formation flight and inter-satellite communication have to be resolved beforehand. In addition, the fractionated modules for interplanetary missions are also required to operate synchronously and fly nearby, spontaneously raising the difficulties of their launch and deployment.

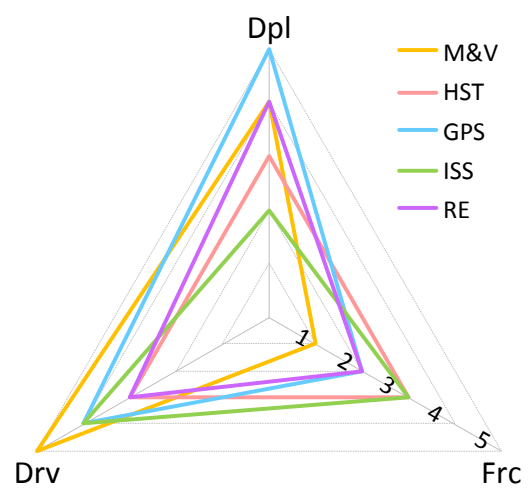

Fig. 7: Comparisons of Different Mission Characteristics in the System Characteristic Space

HST is a space telescope launched into LEO for deep space observation, which has experienced repairing, updating and replacement throughout four Space Shuttle missions, and costs at least $\$ 5$ billion in FY 2010 dollars over the last 25 years[36]. This indicates that Hubble also has some derivation degree, although as a monolithic configuration. On the other hand, such a huge maintenance cost may indicate that monolithic architecture is obviously not an economic solution at least for derivation.

Assuming Hubble is designed in a distributed structure, it is believed that the lifecycle servicing cost will decrease as its fractionation degree increases. For instance, the servicing mission $3 \mathrm{~A}$ was to replace six gyroscopes, a fine guidance sensor and the main computer[37, 38], if such subsystems as command \& data handling subsystem were spatially separated from the payload, only a few new subsystems would be launched and huge maintenance cost could be saved, regardless of the testing of on-orbit servicing technology. This indicates the outstanding flexibility and robustness in long lifecycle missions, derived from distributed configurations. In other words, increasing the degree of fractionation and duplication of a space system is beneficial for reducing the lifecycle cost, with the considerations of on-orbit servicing such as repairing, replacement and updating.

The well-known GPS is a space-based navigation system providing location and time information in various weather conditions. The overall configuration of GPS is considered to be identical, for satellites in the same block performing the same functionality, can be simply replaced by each 
other. Satellites among different blocks also can be considered as functionally identical, since they implement the same function but manifest different behaviours. Such a difference between new blocks and old blocks can be classified as derivation in an identical space system, that is, new blocks retain the same function of old ones, but have some updates and reinforcements, e.g., GPS block IIIA aimed to prevent technical mistakes still threatening GPS block IIF, in addition to achieving some more ambitious goals $[39,40]$. Last but not the least, such a homogeneous configuration indeed makes GPS lasting for a long lifetime, compared with any monolithic navigation satellite.

The US GPS, associated with the Russian GLONASS, the European Galileo, makes up the Global Navigation Satellite System (GNSS). As to functionality, each system is near identical, despite different system performances. Furthermore, all of them can cooperate to implement more ambitious missions. Conceptually, these systems are unlikely to be derivative from each other, although each one can be derivative from itself.

The situation of ISS is a bit different. In terms of system functionality, it has a modular configuration, with varieties of functionalities fractionated. However, all the modules are docked, leaving the entire system as a monolith. Therefore, ISS can be considered as the origin of fractionation, which is also the boundary of monolith.

The monolithic configuration of ISS has its own advantages and disadvantages. On one hand, all the capsules or modules are docked together, so that wireless communication devices and other mass penalty caused by fractionation can be avoid naturally. On the other hand, components to be launched have to possess the ability of rendezvous and dock, which inevitably increases the cost and complexity of the maintenance and development of a space station. Moreover, both scalability and updatability are largely limited by the initial design of the system, which restricts the architecture adjustment and technical advancement. If the space station was designed in a distributed architecture, this mission might be completed in a more simple and cooperative way. It is because most capsules or modules can be designed separately without any docking ports, saving a lot of cost and time among different space agents. Therefore, reconfiguration and upgrades can be realised, even wireless communication framework is possible to be changed to some extent.

RapidEye refers to a constellation of five identical observation satellites, which is operated by BlackBridge AG, providing geospatial information for decision making. The configuration of RapidEye is no doubt to be identical, since all the satellites are equally deployed in the same orbital plane, containing the same sensors[41, 42]. Different from the above constellations, RapidEye is comprised of small and inexpensive platforms with COTS componets. This indicates that tailored and complicated structures can be replaced by modular and standardlised designs, which brings in additional derivation.

\section{Configuration Design Architecture}

In accordance with the qualitative understanding of system configuration tradespace, the general philosophy of value-centric design will be illustrated, based on which the configuration design architecture will be proposed. Overall, this proposed value-centric architecture acts as the systematic guideline of the quantitative design and analysis, with the specific mathematical approaches and techniques described to formulate each procedure of the architecture.

\subsection{System Configuration Design Architecture}

To enable the application of optimization methods and improve the system capabilities and performance with traditional or innovative concepts and technologies, a new value-centric design architecture based upon the system characteristic tradespace is proposed, with each step specifically described as follows:

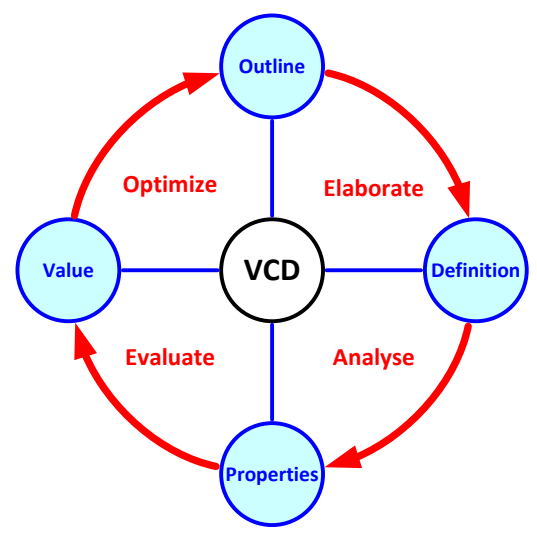

Fig. 8: Value-Centric Design (VCD)

(1) Elaborate. Initially, the rough outline of the system to be designed is parameterized by the design variables of system configuration, namely the degree of duplication, fractionation and derivation. In the Elaborate process, these design variables are transformed into the complete definition of the overall system configuration in the subsystem level. Therefore, the key technique of this step is the description or formulation of the proposed system characteristic space and the overall system configuration definition, as well as their transformation relations.

(2) Analyse. In the analysis process, the relationships between the internal system configuration and the external system properties are established. The integration of subsystem properties into system properties are divided into two steps: the first is to establish the subsystem property models; the second is to discover the integration philosophy by exploring the space system configuration characteristics. As a generic design architecture, such a system configuration definition method should enable the use of monolithic, duplicated, fractionated configurations or any of their feasible combinations. 
(3) Evaluate. In the evaluate process, the overall system value is calculated by appropriate system value models to determine whether the system requirements are met, based on different system properties. As the major element distinguishing the value-centric design from the traditional one, the value model is the enabling factor of the optimization of the concept design process, while the technique difficulties reside how to measure different system properties in the same dimension. One of the possible approaches is to transfer them into the dimension of cost, however the cost characteristics of some system properties are currently unclear.

(4) Optimize. In the optimize process, system value is quantified as objective function or the output of the single design loop, which is returned to design variables as feedback. Such a data flow loop distinguishes the value-centric design from the human-in-the-loop of the requirementcentric design. Under the proposed value-centric design architecture, the optimization process is realised by optimization algorithms rather than the manual modification. Therefore, the theoretical optimal configuration design can be achieved, instead of reaching an empirically feasible solution to meet the mission requirements. In conclusion, the proposed system configuration design architecture has been established based upon the value-centric philosophy. The aim of enabling the use of both traditional and innovative design concepts and technologies has been accomplished theoretically, with the specific approaches and techniques described in the following sections.

\subsection{Formulation of System Characteristic Space}

Assuming $m$ satellites in a space system, and $n$ types of subsystems in each satellite, in this case, the elements of the System Design Matrix $D_{k}$ are the particular property, $k$, of every subsystem on every satellite. Where each element $d_{i j}(i=1,2, \cdots, m, j=1,2, \cdots, n)$ denotes the value of that type of subsystems property on that particular satellite. For example, for the property "mass", the value of element $d_{1,2}$ is the total mass of all subsystems of type 2 on satellite 1 . There is a separate $D$ matrix for each relevant property, e.g. mass, cost, reliability, etc.

$$
D=\left[\begin{array}{cccc}
d_{11} & d_{12} & \ldots & d_{1 n} \\
d_{21} & d_{22} & \ldots & d_{2 n} \\
\vdots & \vdots & \ddots & \vdots \\
d_{m 1} & d_{m 2} & \ldots & d_{m n}
\end{array}\right]
$$

This system design matrix can be understood in two dimensions: on one hand, each row defines the different designs of these $n$ subsystems in a certain satellite; on the other hand, each column displays the distribution of certain subsystem in the system or across the satellites.

For additive system properties such as mass and cost, each element $d_{i j}$ is simply the product of the unit property of that subsystem times the degree of duplication. For non-additive properties, such as reliability, a more sophisticated function of degree of duplication is used.

$$
d_{i j}=n_{i j} u_{i j}
$$

Where, $n_{i j}$ and $u_{i j}$ are the degree of duplication and the subsystem unit design property of subsystem $j$ in satellite $i$ respectively. Let

$$
\begin{array}{r}
N=\left[\begin{array}{cccc}
n_{11} & n_{12} & \ldots & n_{1 n} \\
n_{21} & n_{22} & \ldots & n_{2 n} \\
\vdots & \vdots & \ddots & \vdots \\
n_{m 1} & n_{m 2} & \ldots & n_{m n}
\end{array}\right] \\
U=\left[\begin{array}{cccc}
u_{11} & u_{12} & \ldots & u_{1 n} \\
u_{21} & u_{22} & \ldots & u_{2 n} \\
\vdots & \vdots & \ddots & \vdots \\
u_{m 1} & u_{m 2} & \ldots & u_{m n}
\end{array}\right]
\end{array}
$$

Where, we call $N$ the System Configuration Matrix, defining the number of identical or near identical subsystems in each satellite, or the degree of duplication of each subsystem in each satellite. Once the System Configuration Matrix $N$ is determined, the system configuration can thereby be determined. The Unit Design Property Matrix $U$ can be used for system property analysis to represent any kind of property of each subsystem conceptual design. Therefore

$$
D=N \circ U
$$

\subsubsection{Duplication, Fractionation and Sequence}

Mathematically, degree of duplication can be defined as the number of identical or near identical subsystems scattered in the overall system, according to its definition. Therefore, the System Duplication Vector $P$ can be calculated from the System Configuration Matrix $N$.

$$
p=\left[\begin{array}{llll}
p_{1} & p_{2} & \ldots & p_{n}
\end{array}\right]^{T}
$$

Where, each $p_{j}$ is the sum of elements in each column of $N$.

$$
p_{j}=\sum_{i=1}^{m} n_{i j}
$$

Similarly, the System Fractionation Vector $F$ can be mathematically determined by the number of different satellites with the same certain subsystem.

$$
F=\left[\begin{array}{llll}
f_{1} & f_{2} & \ldots & f_{n}
\end{array}\right]^{T}
$$

Where, each $f_{j}$ is the number of nonzero elements in each column of $N$.

It is obvious that the System Duplication Vector $P$ and the System Fractionation Vector $F$ can be derived from the System Configuration Matrix $N$, called forward transformation. However, the System Configuration Matrix $N$ cannot be derived only from the System Duplication Vector $P$ and the System Fractionation Vector $F$. To make 
this transformation reversible, the System Sequence Vector $Q$ is introduced to complement the information lost during the forward transformation.

$$
Q=\left[\begin{array}{llll}
q_{1} & q_{2} & \ldots & q_{n}
\end{array}\right]^{T}
$$

Where, each $q_{j}$ is the sequence number of each subsystem permutation in the overall system, determined by its ranking in the Subsystem Arranging Matrix $A_{j}$.

$$
A_{j}=\left[\begin{array}{cccc}
a_{j, 11} & a_{j, 12} & \ldots & a_{j, 1 n} \\
a_{j, 21} & a_{j, 22} & \ldots & a_{j, 2 n} \\
\vdots & \vdots & \ddots & \vdots \\
a_{j, m 1} & a_{j, m 2} & \ldots & a_{j, m n}
\end{array}\right]
$$

Where, $A_{j}$ is the Subsystem Arranging Matrix of subsystem $j$, and $n_{j}$ is the corresponding total number of possible arrangements. The calculation process of $n_{j}$ can be considered as one of the classical problems in combinatorics, $p_{j}$ unlabelled ball in $f_{j}$ labelled buckets.

$$
n_{j}=\left(\begin{array}{c}
f_{j} \\
m
\end{array}\right)\left(\begin{array}{l}
f_{j}-1 \\
p_{j}-1
\end{array}\right)
$$

Moreover, all the Subsystem Arranging Matrices $A_{j}$ together constitute the System Arranging Matrix $A$.

$$
A=\left[\begin{array}{llll}
A_{1} & A_{2} & \ldots & A_{n}
\end{array}\right]
$$

The System Arranging Matrix $A$ is derived by the System Duplication Vector $P$ and the System Fractionation Vector $F$. Once given the System Duplication Vector $P$ and the System Fractionation Vector $F$, all the possible arrangements of the system configuration can be determined, and the System Arranging Matrix $A$ is the assembly of these permutations. Meanwhile, these arrangements are sorted from largest to smallest, column by column, in the System Arranging Matrix $A$.

Therefore, the System Duplication Vector $P$ and the System Fractionation Vector $F$, associated with the System Sequence Vector $Q$, have established a one-to-one correspondence with the System Configuration Matrix $N$, and the transformation from $P, F$ and $Q$ to $N$ can be called backward transformation, namely, the reversible transformation of the forward one.

For instance, assume that $m=6, n=5$, and the System Configuration Matrix $N$ is as follows

$$
N=\left[\begin{array}{lllll}
1 & 0 & 0 & 0 & 0 \\
0 & 2 & 0 & 0 & 0 \\
1 & 2 & 1 & 0 & 0 \\
0 & 0 & 1 & 0 & 0 \\
0 & 0 & 0 & 1 & 1 \\
0 & 0 & 0 & 0 & 2
\end{array}\right]
$$

Through the forward transformation, we can obtain the System Duplication Vector $P$ and the System Fractionation Vector $F$ as follows

$$
P=\left[\begin{array}{lllll}
2 & 4 & 2 & 1 & 3
\end{array}\right]^{T}
$$

$$
F=\left[\begin{array}{lllll}
2 & 2 & 2 & 1 & 2
\end{array}\right]^{T}
$$

Take the sequence calculation of subsystem $j=2$ as an example. Since $p_{2}=4$ and $f_{2}=2$, the Subsystem Arranging Matrix $A_{2}$ can be derived with the values below.

$$
A_{2}=\left[\begin{array}{cccccc}
3 & 3 & \ldots & 0 & \ldots & 0 \\
1 & 0 & \ldots & 2 & \ldots & 0 \\
0 & 1 & \ldots & 2 & \ldots & 0 \\
0 & 0 & \ldots & 0 & \ldots & 0 \\
0 & 0 & \ldots & 0 & \ldots & 1 \\
0 & 0 & \ldots & 0 & \ldots & 3
\end{array}\right]
$$

Where

$$
n_{2}=\left(\begin{array}{l}
f_{2} \\
m
\end{array}\right)\left(\begin{array}{l}
f_{2}-1 \\
p_{2}-1
\end{array}\right)=45
$$

It is worth noting that the $20^{\text {th }}$ column vector of $A_{2}$ matches the configuration of subsystem $j=2$, that is, the $2^{\text {nd }}$ column vector of $N$, namely

$$
A_{2}(20)=N(2)
$$

Where, $A_{j}(k)$ and $N(k)$ are the $k^{\text {th }}$ column vector of the matrices $A_{j}$ and $N$ respectively. Thus, we can conclude that $q_{2}=20$. Similarly, the value of the other elements of the System Sequence Vector $Q$ can also be calculated.

$$
Q=\left[\begin{array}{lllll}
2 & 20 & 10 & 5 & 30
\end{array}\right]^{T}
$$

The above exemplifies the forward transformation, while the following is to implement the backward transformation. Since matrices $P, F$ and $Q$ are defined, as the derivative of $P$ and $F$, the System Arranging Matrix $A$ is also available. Therefore, the System Configuration Matrix $N$ can be deduced by column vectors of matrix $A$.

$$
N=\left[\begin{array}{lllll}
A_{1}(2) & A_{2}(20) & A_{3}(10) & A_{4}(5) & A_{5}(30)
\end{array}\right]^{T}
$$

\subsubsection{Derivation}

To achieve the mathematical definition of derivation, the relationships of subsystems in different satellites need to be investigated first, that is, the relationships of the elements in the same column of matrix $U$.

Start from one of the simplest conditions that each subsystem adopts the same design paradigm or the same COTS component, so that all the elements of certain subsystem design option, which are in the same column of matrix $U$, can be considered as identical.

$$
u_{i j}=u_{j}(i=1,2, \cdots, m)
$$

Or

$$
U_{c}=\left[\begin{array}{cccc}
u_{1} & u_{2} & \ldots & u_{n} \\
u_{1} & u_{2} & \ldots & u_{n} \\
\vdots & \vdots & \ddots & \vdots \\
u_{1} & u_{2} & \ldots & u_{n}
\end{array}\right]
$$


For the condition that subsystems differ from each other in different satellites, the System Derivation Matrix is introduced, which defines the degree of derivation of each subsystem in each satellite.

$$
V=\left[\begin{array}{cccc}
v_{11} & v_{12} & \ldots & v_{1 n} \\
v_{21} & v_{22} & \ldots & v_{2 n} \\
\vdots & \vdots & \ddots & \vdots \\
v_{m 1} & v_{m 2} & \ldots & v_{m n}
\end{array}\right]
$$

Where, each $v_{i j}$ presents the degree of derivation in percentage for each subsystem in each satellite. The degree of derivation is similar to the Heritage Factor discussed in the Space Mission Analysis and Design[34], defined as the percentage of a subsystem that is identical to previous spacecraft by mass. Therefore, the System Derivation Matrix $V$ denotes the recurring characteristic of subsystem design from previous ones, which might have significant impacts on system properties, such as reliability.

$$
U=V \circ U_{c}
$$

For real and complex cases like GPS and Galileo, a series of System Derivation Matrices can be applied to exhibit the evolution of the design of satellites.

$$
U=\sum_{k=1}^{n_{g}} V_{k} \circ U_{k}
$$

Where, $n_{g}$ is the generation number of the satellite design, $U_{k}$ is the Unit Design Property Matrix of generation $k$, and $V_{k}$ is its corresponding System Derivation Matrix.

\subsection{Effects Analysis on Reliability}

Having established the mathematical fundamentals of the system characterisation, this section aims at evaluating the effects of degree of duplication, fractionation, and derivation on the system reliability. Based upon the subsystem property modelling, system characteristics will be analysed from the subsystem level. The integration of system properties will be subsequently implemented to build the mapping relations between system designs and system properties, enabling the analyse and evaluate processes in the proposed value-centric design architecture.

\subsubsection{Subsystem Reliability Models}

Mathematically, reliability is defined by Thompson[43] and Davidson[44] as the probability of a component, device, or system performing its intended function without failure for a specific period of time under nominal conditions. To describe how the failure occurrences are distributed over time, we generally use different types of probability distributions, such as the exponential, Weibull, lognormal, and extreme-value distributions.

Due to its mathematical simplicity, flexibility and applicability to the modelling of different failure behaviours, and the demonstrated ability to fit most lifetime data, the
Weibull distribution[44] is one of the most widely used distributions in reliability analysis. The probability density function (PDF) of the Weibull distribution with a continuous random variable can be written as follows.

$$
r(t)=\left\{\begin{array}{cl}
\left(\frac{\beta}{t}\right)\left(\frac{t}{\eta}\right)^{(\beta-1)} e^{-\left(\frac{t}{\eta}\right)^{\beta}} & t \geq 0 \\
0 & t<0
\end{array}\right.
$$

Where, $t$ is the satellite lifetime in orbit before failure, $\beta$ is the dimensionless shape parameter, $\eta$ is the scale parameter in units of time. Consequently, the reliability cumulative distribution function (CDF) can be expressed as

$$
R(t)=e^{-\left(\frac{t}{\eta}\right)^{\beta}}
$$

This is the reliability model used for the different space subsystems and systems in this paper. For each specific case, the parameters $\beta$ and $\eta$ are calculated by parameter estimation methods, among which Maximum Likelihood Estimation (MLE) and Bayesian theory in combination with Markov Chain Monte Carlo simulations (MCMC) are two of the most effective and applicable. The selection of these two methods is mainly determined by the sample size of the database.

Based on the 1584 Earth-orbiting satellites successfully launched from January 1990 to October 2008 recorded in the SpaceTrak database (STD), Castet and Saleh[45, 46] developed and fitted a parametric reliability model for actual satellite subsystems. Meanwhile, Guo[47] conducted reliability modelling particularly for small satellite subsystems, and found that MCMC is suitable for such databases with small sample sizes and censored data problems as Small Satellite Anomalies Database (SSAD), which covers 222 small satellites launched over the last few decades. It is apparent that SSAD is more applicable for the reliability analysis of small satellite subsystems, while STD might appeal to generic systems.

In Table 1, the estimated Weibull parameters are listed for both STD and SSAD. The abbreviations used here are PLS for Payload Subsystem, EPS for Electrical Power Subsystem, TTC for Telemetry, Tracking and Command Subsystem, CDH for Command and Data Handling Subsystem, ACS for Attitude Control Subsystem, OCS for Orbit Control Subsystem, TCS for Thermal Control Subsystem, and SAS for Solar Array Subsystem.

\subsubsection{Reliability Analysis}

Prior to integrating the system reliability, preliminary effects analyses of duplication and fractionation on independent subsystems are carried out to help understand their impacts on the overall system reliability, using the parameters in Table 1. As shown in Fig. 9, duplicated subsystems enjoy a continuous reliability growth with the increase of the degree of duplication, while fractionated subsystems experience a drop-down and a recovery period, yet still cannot perform as reliable as duplication at the 
Table 1: The Estimated Weibull Parameters per Subsystem[45, 46, 47]

\begin{tabular}{ccccc}
\hline \multirow{2}{*}{ Subsystem } & \multicolumn{2}{c}{ STD } & \multicolumn{2}{c}{ SSAD } \\
\cline { 2 - 5 } & $\beta$ & $\eta$ (years) & $\beta$ & $\eta$ (days) \\
\hline PLS & 0.8874 & 7983 & 0.4162 & 275006 \\
EPS & 0.7460 & 7733 & 0.3110 & 58385 \\
TTC & 0.3939 & 400982 & 0.2588 & 176896 \\
CDH & 0.8874 & 7983 & 0.4162 & 275006 \\
ACS & 0.7182 & 3831 & 0.4144 & 85409 \\
OCS & 0.3375 & 6206945 & - & - \\
TCS & 0.3560 & 21308746 & 0.2655 & 15068200 \\
SAS & 0.4035 & 1965868 & - & - \\
\hline
\end{tabular}

Table 2: Parameters of System Reliability Integration

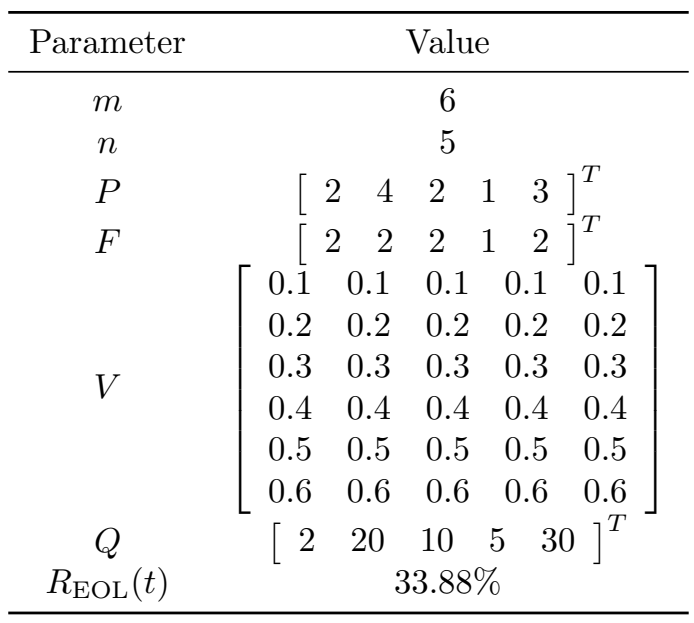

same degree, due to the penalty of extra wireless sharing devices.

Derivation exhibits a different impact on system reliability, by influencing each individual subsystem unit, instead of the entire system configuration. In other words, it assigns different values for each individual element, rather than their combinations.

As Fig. 10 shows, degree of derivation from 0.0 to 1.0 with an interval of 0.1 is represented by varying the colour from the lightest to the darkest. The results reveal that the high heritage factor of mature design ensures a reliable system, while innovative design inevitably brings risks for a system. It is also interesting to note that the reliability increment between the two adjacent curves increases with the increasing heritage factor. In conclusion, derivation provides another insight for system reliability behaviours.

\subsubsection{System Reliability Integration}

Having understood the characteristics of each system configuration vector, the next step is to explore the characteristics of hybrid configurations. By fixing the values of the degree of duplication, fractionation, and derivation, we conduct a simulation of system reliability integration, using the procedure shown in Fig. 11.

The input values used in the simulation and the associ-

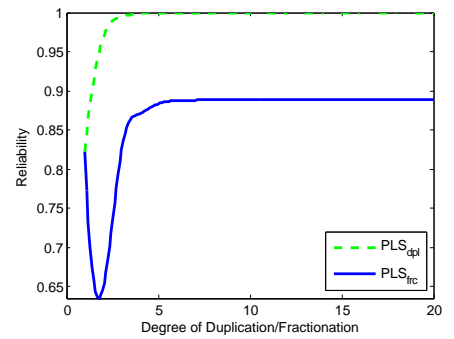

(a) PLS

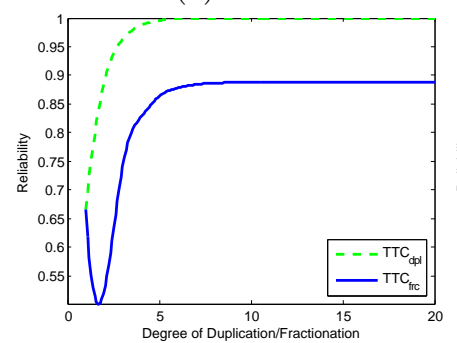

(c) TTC

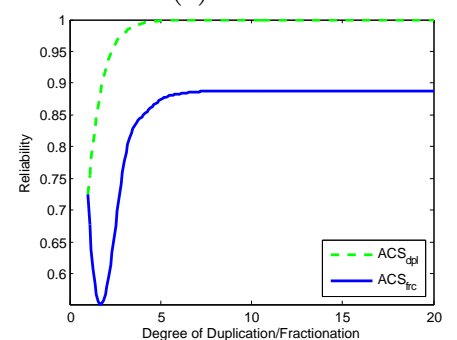

(e) ACS

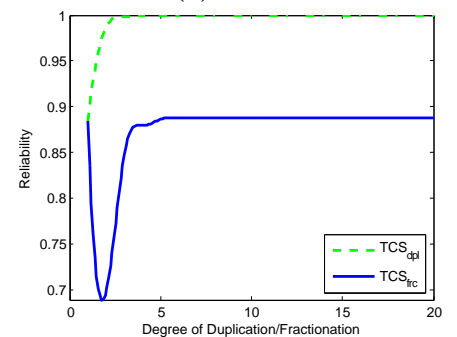

(g) TCS

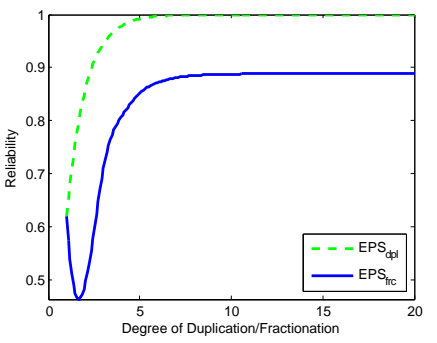

(b) $\operatorname{EPS}$

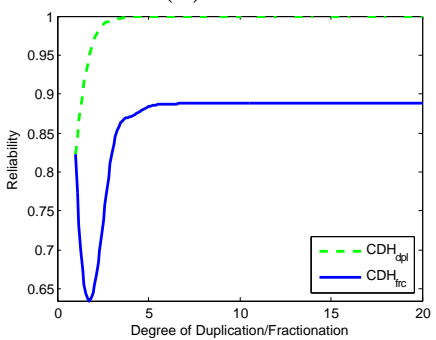

(d) $\mathrm{CDH}$

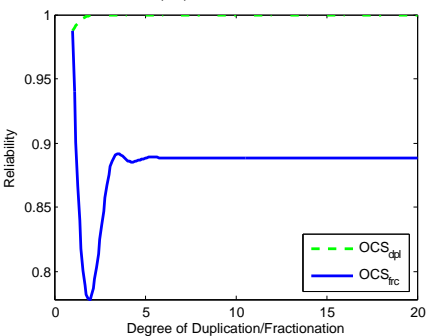

(f) OCS

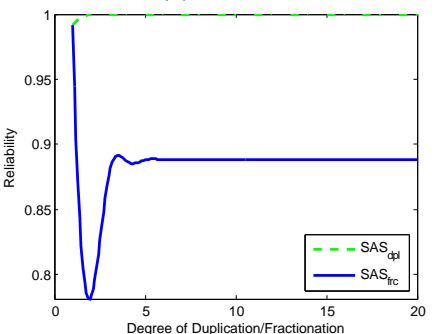

(h) SAS
Fig. 9: Duplication and Fractionation Characteristics per Subsystem

ated output values are listed in Table 2 . Where, $R_{\mathrm{EOL}}(t)$ is the output EOL reliability of the entire system. It is worth noting that the System Sequence Matrix $Q$ is alterable when fixing the vectors of duplication $P$, fractionation $F$, and derivation $V$. The values we set here are random, and the corresponding result is obviously not the optimal permutation for the subsystem resources available, which is the reason why the overall system reliability is not ideal. Therefore, one of potential applications of this research might be to determine the optimal value of $Q$ with fixed $P, F$ and $V$. This would require the use of optimization methods and the connection of the final optimize process for the proposed value-centric design architecture. 


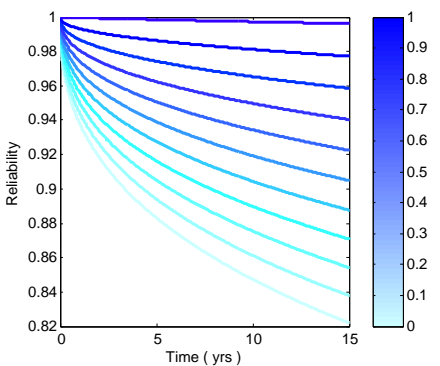

(a) PLS

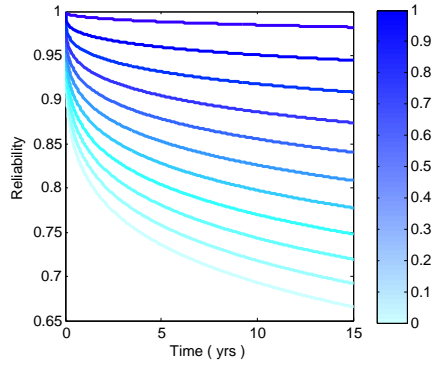

(c) TTC

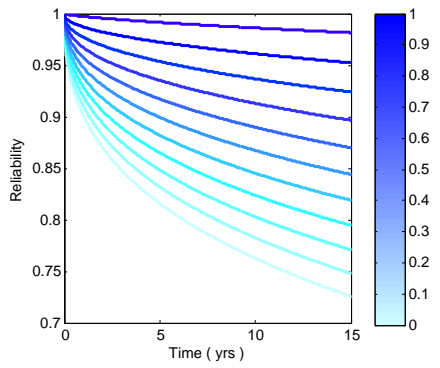

(e) ACS

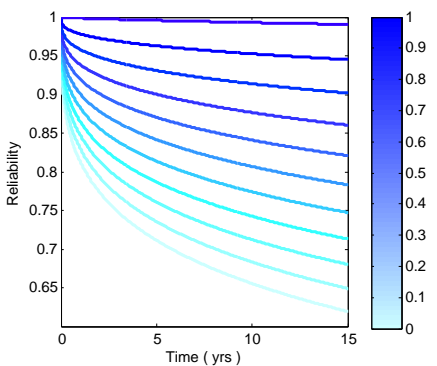

(b) EPS

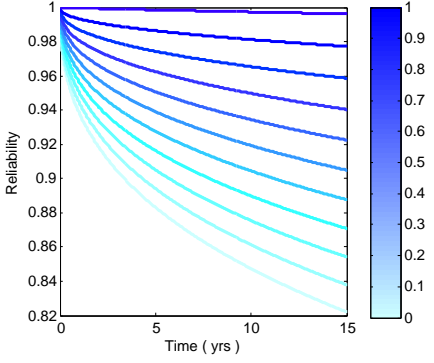

(d) $\mathrm{CDH}$

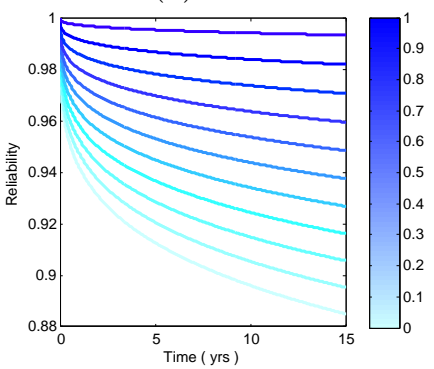

(f) TCS
Fig. 10: Derivation Characteristic per Subsystem (The colourbar indicates degree of derivation)

\section{Conclusion}

Aiming at overcoming the drawbacks inherent in the traditional requirement-centric design philosophy, this paper has developed a new value-centric configuration design architecture to enable the exploration of the effect of emergence of the latest space concepts and technologies.

After the categorisation of various space systems, three configuration characteristics were generalised to construct the system characteristic space. Based on such a space, the connection has been built between mission concept design and system configuration characteristics, enabling the qualitative analysis of the feasibility and applicability of different system configurations for different mission scopes. In other words, a mission concept can be analysed and designed in the system characteristic space, where each dimension reflects different characteristics of the overall configuration. Such a system characteristic perspective may offer designers or users a better understanding of the limits and potentials of a mission, beneficial for mission modification or extension.

In accordance with the definitions of the system characteristic space, the mathematical fundamentals of the

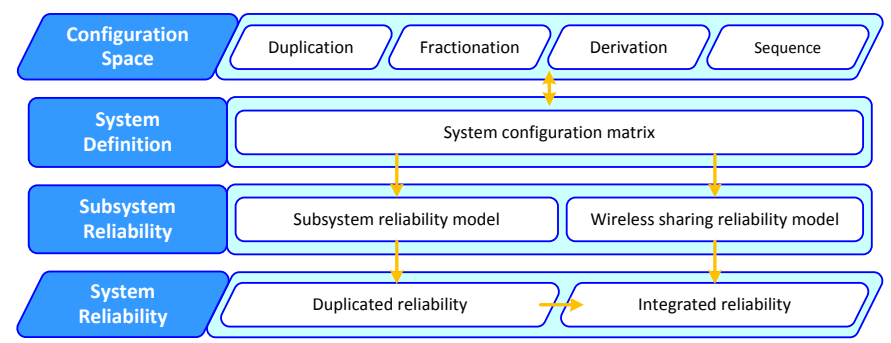

Fig. 11: Integration Procedures of System Reliability

proposed value-centric architecture was developed, establishing the one-to-one correspondence between the overall system configuration matrix and the three dimensions of system characterisation. The mathematical model can improve a given system property by exploring the effect of different subsystem selections, beneficial for the applications of the quantitative analysis and optimization on system designs.

Based on the subsystem property modelling, the effect analyses and simulations have been implemented on different system configuration characteristics to explore the configuration design space. The integration of system properties in the proposed value-centric concept design architecture has been accomplished to build the mapping relations between the system configuration design and the system properties. This may be one of the enabling factors for the applications of optimization methods in the system characteristic space to search for the optimal configuration design, with a system property as the objective function.

The quantification of system values and adoption of optimization algorithms are two major advantages of the proposed value-centric design architecture, which distinguishes it from traditional requirement-centric design methodologies. It enables the design team to design and compare new and innovative architectures on a level playing field. Especially in the era of "smaller, faster, and cheaper" space, the proposed design architecture can provide a vehicle for the introduction of modular, standardised, and/or mass-produced technologies. Thus, helping to address problems such as capability uncertainty, cost overruns, and schedule delays.

\section{Acknowledgements}

The authors gratefully thank the financial support from the China Scholarship Council (CSC) for this work.

\section{References}

[1] A. Poghosyan, A. Golkar, CubeSat Evolution: Analyzing CubeSat Capabilities for Conducting Science Missions, Progress in Aerospace Sciences 88 (September 2016) (2016) 59-83, ISSN 03760421, doi: \bibinfo\{doi\} \{10.1016/j.paerosci.2016.11. 002\}, URL http://dx.doi.org/10.1016/j.paerosci.2016.11. 002. 
[2] P. Davison, D. Kellari, E. F. Crawley, B. G. Cameron, Communications Satellites: Time Expanded Graph Exploration of a Tradespace of Architectures, Acta Astronautica 115 (2015) 442-451, ISSN 00945765, doi:\bibinfo $\{$ doi $\}\{10.1016 /$ j.actaastro.2015.05.017\}, URL http://dx.doi.org/10.1016/j . actaastro.2015.05.017

[3] D. Rossetti, B. Keer, J. Panek, B. Reed, F. Cepollina, R. Ritter, Spacecraft Modularity for Serviceable Spacecraft, in: AIAA SPACE 2015 Conference and Exposition, Pasadena, CA, ISBN 9781624103346, 1-12, URL http://www.scopus.com/inward/record.url?eid=2-s2. 0-84960427990 $\{\backslash \&\}$ partnerID=tZOtx3y1, 2015.

[4] M. Martin, M. Stallard, Distributed Satellite Missions and Technologies - The TechSat 21 Program, in: AIAA Space Technology Conference \& Exposition, Albuquerque NM, 28-30, 1999.

[5] M. D. Graziano, Overview of Distributed Missions, in: M. D'Errico (Ed.), Distributed Space Missions for Earth System Monitoring, chap. 12, New York, ISBN 9781461445401, 375386, doi: \bibinfo $\{$ doi $\}\{10.1007 / 978-1-4614-4541-8\}, 2013$.

[6] C. Mathieu, A. Weigel, Assessing the Flexibility Provided by Fractionated Spacecraft, in: AIAA SPACE 2005 Conference \& Exposition, Long Beach, CA, AIAA 2005-6700, 2005.

[7] S. Nag, L. Summerer, Behaviour Based, Autonomous and Distributed Scatter Manoeuvres for Satellite Swarms, Acta Astronautica 82 (1) (2013) 95-109, ISSN 00945765, doi: \bibinfo\{doi\} $\{10.1016 /$ j.actaastro.2012.04.030\}, URL http://dx.doi.org/ $10.1016 / j$. actaastro. 2012.04.030.

[8] A. Shao, E. A. Koltz, J. R. Wertz, Quantifying the Cost Reduction Potential for Earth Observation Satellites, in: Proceedings of the 12th Reinventing Space Conference, London, ISBN 978-3-319-34023-4, 199-210, doi:\bibinfo $\{$ doi $\}\{10.1007 /$ 978-3-319-34024-1\}, 2014.

[9] N. H. Crisp, K. Smith, P. Hollingsworth, Launch and Deployment of Distributed Small Satellite Systems, Acta Astronautica 114 (2015) 65-78, ISSN 00945765, doi:\bibinfo $\{$ doi $\}\{10.1016 /$ j.actaastro.2015.04.015\}, URL http://dx.doi.org/10.1016/j. actaastro.2015.04.015.

[10] M. T. Hicks, C. Niederstrasser, Small Sat at 30: Trends, Patterns, and Discoveries, in: 30th Annual AIAA/USU Conference on Small Satellites, Logan, UT, 1-13, 2016.

[11] A. Shaw, P. Rosher, Micro Satellites : The Smaller the Satellites , the Bigger the Challenges ?, Air and Space Law 41 (4) (2016) $311-328$

[12] C. Soanes, A. Stevenson, Oxford Dictionary of English, Oxford University Press, Oxford, 2 edn., 2005.

[13] G. F. Dubos, J. H. Saleh, Comparative cost and utility analysis of monolith and fractionated spacecraft using failure and replacement Markov models, Acta Astronautica 68 (12) (2011) 172-184, ISSN 00945765, doi: $\backslash$ bibinfo $\{$ doi $\}\{10.1016 /$ j.actaastro.2010.07.011\}, URL http://dx.doi.org/10.1016/j. actaastro.2010.07.011.

[14] National Aeronautics and Space Administration, Sputnik 1, URL http://nssdc.gsfc.nasa.gov/nmc/spacecraftDisplay. do?id=1957-001B, 2014.

[15] J. C. Cook, D. Agle, D. Brown, NASA Spacecraft Embarks on Historic Journey Into Interstellar Space, URL http://www . nasa.gov/mission\{\_\}pages/voyager/voyager20130912.html, 2013.

[16] National Aeronautics and Space Administration, Earth's Reflection in Dawn Spacecraft, URL http://photojournal.jpl. nasa.gov/catalog/PIA12029, 2009.

[17] European Space Agency, Fact Sheet, URL http://www. spacetelescope.org/about/general/fact $\left\{\backslash_{\_}\right\}$sheet/, 2015.

[18] European Space Agency, Hubble in Orbit, URL http://www . spacetelescope.org/images/hubble\{\_\}in\{\_\}orbit1/, 2011.

[19] J. Guo, D. Maessen, E. Gill, Fractionated Spacecraft: The New Sprout in Distributed Space Systems, in: 60th International Astronautical Congress (IAC), Daejeon, ISBN urn:isbn:9781615679089, 1-11, URL http://repository.tudelft.nl/view/ir/uuid: 43647cd0-9698-4765-a584-a5c087a7ca79/, 2009.
[20] A. Darrin, B. O'Leary, Handbook of Space Engineering, Archaeology, and Heritage, CRC Press, Boca Raton, 2009.

[21] E. Kaplan, C. Hegarty, Understanding GPS: Principles and Applications, Artech House, Norwood, MA, 2 edn., 2005.

[22] National Aeronautics and Space Administration, Tracking and Data Relay Satellite (TDRS) Fleet, URL http://www.nasa.gov/directorates/heo/scan/services/ networks/txt $\left\{\backslash_{\text {_t }}\right\}$ tdrs $\left\{\backslash_{\text {_ }}\right\}$ fleet.html $\{\backslash \#\}$.VVYMlSvF-ig, 2014.

[23] United States Government, GPS Block IIF Satellite, URL http: //www.gps.gov/multimedia/images/IIF \{\\}2.jpg, 2014.

[24] European Space Agency, 30-Satellite Galileo Constellation, URL http://www.esa.int/spaceinimages/Images/2014/ 07/30-satellite $\left\{\backslash_{-}\right\}$Galileo\{ $\backslash$ _ $\}$ constellation, 2014.

[25] C. Mathieu, A. Weigel, Assessing the Flexibility provided by an On-orbit Infrastructure of Fractionated Spacecraft, in: 56th International Astronautical Congress (IAC), Fukuoka, 2005.

[26] C. Mathieu, A. Weigel, Assessing the Fractionated Spacecraft Concept, in: AIAA SPACE 2006 Conference \& Exposition, San Jose, CA, AIAA 2006-7212, 2006.

[27] A. Salado, R. Nilchiani, Fractionated Space Systems: Decoupling Conflicting Requirements and Isolating Requirement Change Propagation, in: AIAA SPACE 2013 Conference \& Exposition, San Diego, CA, 1-15, 2013.

[28] Defense Advanced Research Projects Agency, System F6, URL http://www.darpa.mil/our\{\_\}work/tto/programs/system\{\_ \}f6. aspx, 2015 .

29] O. Brown, P. Eremenko, P. D. Collopy, Value-Centric Design Methodologies for Fractionated Spacecraft: Progress Summary from Phase 1 of the DARPA System F6 Program, in: AIAA SPACE 2009 Conference \& Exposition, Reston, VA ISBN ISSN $\sim 2009-6540$, AIAA 2009-6540, doi: \bibinfo\{doi\} \{AIAA2009-6540\}, 2009.

[30] U.S. Naval Observatory, Current GPS Constellation, URL http://www.usno.navy.mil/USNO/time/gps/ current-gps-constellation, 2016.

[31] C. H. Yinger, Operation and Application of the Global Positioning System, Crosslink 3 (2) (2002) 12-16, URL http: //en.wikipedia.org/wiki/GPS $\left\{\backslash_{-}\right\}$modernization.

[32] O. Brown, P. Eremenko, M. Bille, Fractionated Space Architectures: Tracing the Path to Reality, in: 23rd Annual AIAA/USU Conference on Small Satellites, Logan, UT, 1-10, 2009.

[33] J. Chu, J. Guo, E. K. a. Gill, Fractionated Space Infrastructure for Long-Term Earth Observation Missions, in: IEEE Aerospace Conference, Big Sky, MT, ISBN 9781467318112, ISSN 1095323X, 1-9, doi:\bibinfo $\{$ doi $\}\{10.1109 / A E R O .2013$. $6496854\}, 2013$

[34] J. R. Wertz, W. J. Larson, Space Mission Analysis and Design, Microcosm Press, El Segundo, CA, 3 edn., ISBN 07923590111881883108 (pbk.) 9780792359012 9781881883104 (pbk.), URL http://www.loc.gov/catdir/toc/ fy0703/2005283477.html, 1999 .

[35] O. Brown, P. Eremenko, The Value Proposition for Fractionated Space Architectures, in: AIAA SPACE 2006 Conference \& Exposition, San Jose, CA, 1-22, 2006.

[36] National Aeronautics and Space Administration, James Webb Space Telescope (JWST): Independent Comprehensive Review Panel (ICRP) Final Report, Tech. Rep., National Aeronautics and Space Administration, Washington, DC, 2010.

[37] National Aeronautics and Space Administration, SM3A, URL http://web.archive.org/web/20080418092326/http:// hubble.nasa.gov/missions/sm3a.php, 2006.

[38] Lockheed Martin, Hubble Space Telescope Servicing Mission 3A - Media Reference Guide, Tech. Rep., Lockheed Martin, 1999.

[39] United States Government Accountability Office, GLOBAL POSITIONING SYSTEM: Significant Challenges in Sustaining and Upgrading Widely Used Capabilities, Tech. Rep., United States Government Accountability Office, Washington, DC, 2009.

[40] United States Government Accountability Office, GLOBAL POSITIONING SYSTEM: Challenges in Sustaining and Up- 
grading Capabilities Persist, Tech. Rep., United States Government Accountability Office, Washington, DC, 2010.

[41] A. M. Baker, B. Stocker, J. Gebbie, M. Oxfort, G. Tyc, J. Steyn, N. Hannaford, RapidEye - A cost-effective Earth Observation Constellation, in: 59h International Astronautical Congress (IAC), Glasgow, 2008.

[42] J. Gebbie, P. Davies, A. d. S. Curiel, G. Tyc, L. Boland, P. Palmer, Spacecraft Constellation Deployment for the RapidEye Earth Observation System, in: 60th International Astronautical Congress (IAC), Daejeon, 2009.

[43] G. Thompson, Improving Maintainability and Reliability through Design, Professional Engineering Publishing, London, 1999.

[44] J. Davidson, The Reliability of Mechanical Systems, Mechanical Engineering for the Institution of Mechanical Engineers, London, 1988.

[45] J.-F. Castet, J. H. Saleh, Satellite Reliability: Statistical Data Analysis and Modeling, Journal of Spacecraft and Rockets 46 (5) (2009) 1065-1076.

[46] J.-F. Castet, J. H. Saleh, Satellite and Satellite Subsystems Reliability: Statistical Data Analysis and Modeling, Reliability Engineering and System Safety 94 (11) (2009) 1718-1728, ISSN 09518320, doi: \bibinfo $\{$ doi $\}\{10.1016 /$ j.ress.2009.05.004\} .

[47] J. Guo, L. Monas, E. Gill, Statistical Analysis and Modelling of Small Satellite Reliability, Acta Astronautica 98 (1) (2014) 97-110, ISSN 00945765, doi: bibinfo\{doi $\}$ 10.1016/j.actaastro. 2014.01.018\}, URL http://dx.doi.org/10.1016/j.actaastro. 2014.01.018. 